\title{
The hormones involved and the prevention of fecal and urinary urge incontinence
}

\author{
Walter G Roper* \\ 21/33 Bernard Rd., Padstow Heights, Sydney, NSW, Australia
}

\begin{abstract}
This paper is of critical importance throughout the world. It shows how both urinary urge and fecal urge incontinence can be largely prevented provided the sufferers abide by an imposed diet which is not too restrictive and in fact, is healthy. Huge saving in cost of health care (largely in the aged) will be made if this diet is taken up widely.
\end{abstract}

In the past 50 years, life expectancy has increased by 11 years. This means the former 75 years of age is now 86 years which makes a significant difference to continence. The latter is maintained by the anal sphincters. There are two of these, the External Sphincter which is under voluntary and quasi-voluntary nervous control (by the pudendal nerve which carries somatic motor and sensory fibers) and the Internal Sphincter which lies underneath it and is entirely involuntary. It is innervated by sympathetic fibers from the superior rectal and hypogastric plexuses. Its contraction is inhibited by parasympathetic nervous stimulation. It is normally in a state of tonic closure due to its sympathetic innervation which causes it to undergo 8-30 phasic contractions per minute [1]. These are responsible for $85 \%$ of its closure and the remaining control is by the external sphincter.

The internal anal sphincter is also innervated by the parasympathetic nervous system (S2-4) which is in direct opposition to the sympathetic influence and will cause the sphincter to open once it overcomes the latter and the external sphincter allows this to happen by voluntary command. During the ageing process, there is a decline in power of both the sympathetic and the parasympathetic forces. This affects the sympathetic more because of its greater activity and the result is a degree of weakening of the overall closure power of the anus. Apart from the sympathetic and parasympathetic sections of the autonomic system, there is a third division called the Enteric nervous system (ENS) [2], which consists of some 500 million neurons which is 5 times the number in the spinal cord. It extends from the oesophagus right through to the anus. The neurons are collected in 2 types of ganglia- the myenteric (Auerbach's), located between the inner and outer layers of the muscuralis externa, and the submucosal (Meissner's) located in the submucosa.

Meissner's plexus consists of weak afferent fibers in the submucous zone which advise the brain when defaecation is needed. There are both circular and longitudinal muscles in the intestines. The longitudinal muscle contracts proximally causing obstruction and dilates distally resulting in peristalsis while the circular muscle opens at both ends causing mixing of intestinal contents (chyme) for better absorption. The plexus innervates the muscle with parasympathetic and sympathetic supply. The enteric nervous system is capable of autonomous function by running the intestines on its own but has connections to both the major sympathetic and parasympathetic systems which guide it and make it run much more smoothly. The system makes use of more than 30 neurotransmitters such as acetyl choline, dopamine, and serotonin.

When sucrose or glucose is ingested, the enteric system is stimulated and secretes parasympathetic but also sympathetic hormone. The effective result is parasympathetic, and this is necessary to aid the maturation processes necessary in the Islets of Langerhans to allow the prepre and pre changes to take place, which is essential before the Beta cells of the pancreas can secrete insulin. The complexity of the interacting neurons is enormous and not fully understood yet especially since there is plasticity which enables the sudden changing of sympathetic to parasympathetic effect and vice versa. This makes understanding the integration of the systems too difficult to understand at this stage of research. To overcome this, it is necessary to be guided by the usually constant result of the various processes at hand. Fecal Urge Incontinence means that when the urge to defecate occurs, the person involved, senses the urgency but does not have sufficient time to get to a toilet and mass emptying of the rectum takes place. The action is usually explosive and the faeces are usually liquid or semi solid. Under normal conditions the rectum has enough time to extract water from them and the rectum empties in sections thereby dissipating the high pressure within. Following the above mass rectal emptying, defecation quickly reverts to normal. This whole process takes place when there is sufficient parasympathetic hormone to overcome the sympathetic supply and cause mass contraction of the rectum. The subsequent mass evacuation of faeces is withheld by the voluntary contraction of the external anal sphincter but not for long before it gives way.

There is a threshold for the amount of parasympathetic hormone required and this coincides with the threshold for the amount of sucrose or glucose which can be ingested, and this increase may, by

${ }^{\star}$ Correspondence to: Walter Geoffrey Roper, 21/33 Bernard Rd., Padstow Heights, Sydney, NSW, Australia, Tel: 0415570 202; E-mail: w.roper@bigpond.com

Key words: urge, incontinence, hormones, neurology

Received: June 12, 2020; Accepted: July 06, 2020; Published: July 10, 2020 
addition, take place gradually during the day because of further sucrose ingestion These thresholds are lower in the elderly because of the lower amount of sympathetic hormone which is keeping their anal sphincters closed.

The condition of urge incontinence is more common in women and this is largely due to damage done to their anal sphincters during childbirth. Surgical damage (eg from hemorrhoidectomy) can also be a factor in both sexes but is much less common. Once the threshold for glucose ingestion has been reached, it can be very quick for the rectum to react strongly, indicating that the parasympathetic hormone involved, has been carried there in the blood. Vagal innervation of the stomach and intestines ends mid colon and is not the cause of this condition. The conduct of food from the stomach to the anus takes about 2 days. Because of the huge embarrassment which occurs with this incontinence, the sufferers rarely talk about it and often won't even tell their doctor and many turns into recluses. This is a great pity since we believe it can be prevented by appropriate diet. Apart from the ingestion of sugar and other disaccharides, other factors must be considered. These are coffee, alcohol, and any other substances which might have a dilatory action such as theophylline in tea.

Urinary urge incontinence must be considered here because it is so similar to the fecal type, with internal and external sphincters with the same type of innervation and structure. Similar diet can apply to both, but urinary incontinence must be treated with pelvic floor exercises also. In the early stages, fecal incontinence can also be helped by such exercises. These are best carried out by pulling up the anus and then releasing it. This action should be repeated about 5 times in succession. Each time it is pulled up, it should be held up for 5 seconds and when relaxed, for 4 seconds. This action automatically affects the urinary sphincter also, but more gently and without side effects. In urinary urge incontinence, it needs to be kept up for a much longer overall period than in fecal urge incontinence.

\section{Diet}

To start, it is wise to gradually reduce coffee until it is eliminated. This removal must be maintained in urinary urge incontinence. Caffeine is a diuretic causing the bladder to fill more frequently and it also weakens the constrictor power of the urinary sphincters. Sugar must also be largely eliminated at first because its ingestion is the main problem by stimulating the enteric cells to secrete parasympathetic hormone. In fecal incontinence, there is a threshold involved but in the early diet stages it is wise to stay well below this until appropriate body adjustment occurs.

Fruits must be carefully rationed in urge incontinence not because of its fructose content which does not cause insulin secretion and is not a factor in this paper's theme. Fructose can cause fatty liver leading to the metabolic syndrome and then to type 2 diabetes which, because of diet mixture with sweet corn syrup, is almost epidemic in the USA. In the urge incontinence syndrome, it is purely sucrose content which is of critical importance and the highest amount of this, is found in grapes, mango, kiwifruit, orange, passionfruit and bananas and the amount of this fruit ingested must be carefully monitored. After several months, during which continence is retained, it is possible to add subthreshold items to. The diet, such as a morning cup of coffee and one glass of alcohol for women and two for men without any risk at all. Fruit must still be rationed especially that with high sucrose content (a whole mango can never be permitted, and the number of high sucrose foods strongly limited). The frequency of urge incontinence in aged care and retirement villages is very high and stopping this will mean far less work for the amazing nursing staff who take care of it. Much money will be saved also.

\section{Urinary urge incontinence}

This is the same as in Fecal Urge Incontinence except that no coffee or tea is ever allowed, and alcohol limited to one glass (but no beer). Also, pelvic floor exercises must be continued.

Fecal Urge Incontinence: Fruit must be strictly limited to 2 amounts per day.

These amounts are:

Mango-not more than I quarter. (can serve as 1 amount); Apple-a quarter only. (can serve as 1amount); Banana-1 half; Cherries-8; Grapes-4; Kiwi fruit-1 small or half a larger one; Berries-5; 1 of the following-pear, orange, mandarine, apricot; Watermelon -1 small quarter slice; Rockmelon-2 cubes' Bread - limit daily amount; Rice limit daily white or brown rice

High Protein Foods: lean meat, chicken (no skin), eggs are allowed and encouraged.

Milk-lactose free, is preferred.

Cheese: equivalent to 1 slice daily.

Green tea: 1 cup daily (infused for 2.5 minutes)

Fats: are best kept low.

Artificial sweeteners: Sorbitol and some others are best avoided [3]. Only the colon can digest

sorbitol and it attracts water and can cause osmotic diarrhoea.

Vegetables: 4 are allowed per day.

Sweet potato microwave- cooked preferable but steamed allowed but not baked.

Potato- please limit to low quantity.

The following are alright: asparagus, avocado, artichoke, broccholi, cabage, cauliflower, celery, cucumber, eggplant, lettuce, mushrooms, tomatoes, onions, spinach, zucchini, turnips, green peas and beans.

These are all glycemic index low food (Glycemic Index Foundation) but the quantity must be no more than one serving.

Alcohol: 1 glass of wine for women and 2 for men are allowed.

The effect of excess alcohol depends on the health of the recipient. It can cause insulin secretion and hence this condition, but usually only if the amount taken is in the danger zone, which is over 4 glasses. Tolerance to alcohol develops and leads many people to take higher and higher amounts. This in turn leads to destruction of theta cells in the pancreas stopping any insulin production. Excess alcohol can cause great damage to the brain and can cause peripheral neuritis and damage of the autonomic nervous system including the enteric nervous system. This means that its neurons produce much less parasympathetic hormone than usual. It can cause diarrhoea by increasing the fluid in the intestines [4] but this diarrhoea is much slower to occur usually and continues for a total of about 3 days, unlike fecal urge incontinence. This is why trials with alcohol excess often end with opposite results depending on the health of the recipient as stated. 


\section{References}

1. Bhardwaj R, Vaizey CJ, Boulos PB, Hoyle CH (2000) Neuromyogenic properties of the internal anal sphincter. Gut 46: 861-868.

2. Furness JB (2007) Enteric nervous system. Scholarpedia 2: 4064
3. Hyams JS (1982) Osmotic diarrhoea with sorbitol. Section of Pediatric gastroenterology, Hartford Hospital, Hartford, Connecticut.

4. https://www.healthline.com/health/alcohol-poop\#1

Copyright: (C2020 Roper WG. This is an open-access article distributed under the terms of the Creative Commons Attribution License, which permits unrestricted use, distribution, and reproduction in any medium, provided the original author and source are credited. 